\title{
On the Law of Large Numbers for Discrete Fourier Transform
}

\author{
Na Zhang \\ University of Cincinnati, PO Box 211005, Cincinnati, OH 45221-0025 \\ Email: zhangn4@mail.uc.edu
}

\begin{abstract}
We shall establish the law of large numbers for the discrete Fourier transform of random variables with finite first moment under condition $P\left(\left|X_{n}\right|>x\right) \leq P\left(\left|X_{1}\right|>x\right)$ for all $x \geq 0$; for $1<p<2$, we establish the Marcinkiewicz-Zygmund type rate of convergence for the discrete Fourier transform of random variables with finite $p t h$ moment under condition $\frac{1}{n} \sum_{k=1}^{n} P\left(\left|X_{k}\right|>\right.$ $x) \leq M P\left(\left|X_{1}\right|>x\right)$ for all $x \geq 0$ and some positive constant $M$.
\end{abstract}

Key words: Law of large numbers; Discrete Fourier transform; Rate of convergence Mathematical Subject Classification(2010): Primary 60F15, secondary 42A20

\section{Introduction}

The law of large numbers is valid for pairwise independent identically distributed random variables, result due to Etemadi (1981). This is a surprising result since a sequence of pairwise independent identically distributed random varibles may not be ergodic. A way to look into the speed of convergence of this result when the variables have finite moments of order $r, 1<r<2$, is provided by Baum and Katz (1965) in the i.i.d. case and by Stocia (2011) in the martingale difference case. By carefully examining the proof in Stoica (2011), we notice that the proof can be adapted to centered pairwise independent random variables and we can formulate the following result.

Proposition 1.1. Let $\left(X_{n}\right)_{n \geq 1}$ be a pairwise independent sequence of random variables with the same distribution.

(a) Assume $X_{n} \in L^{1}$. Then

$$
\frac{S_{n}}{n} \rightarrow E X_{1}, \quad P-\text { a.s. as } n \rightarrow \infty
$$

where $S_{n}=\sum_{k=1}^{n} X_{k}$.

(b) Assume $X_{n} \in L^{p}, 1<p<2$. Then for all $1 \leq r \leq p$ and $\epsilon>0$,

$$
\sum_{n=1}^{\infty} n^{p / r-2} P\left(\left|S_{n}\right|>\epsilon n^{1 / r}\right)<\infty .
$$

We note that, by Korchevsky (2015), $\frac{S_{n}-E S_{n}}{n^{1 / p}} \rightarrow 0, \quad P-$ a.s. as $n \rightarrow \infty$, also holds . 
The goal of our note is to study the law of large numbers for the discrete Fourier transform of a sequence of random variables with finite $p t h$ moment for $p=1$ and its Marcinkiewicz-Zygmund type rate of convergence for $1<p<2$ under weaker conditions than identical distribution as described in the abstract and to show that, from some point of view, the variables have similar properties as pairwise independent random variables with the same distribution.

\section{$2 \quad$ Main Results}

Let $\left(X_{n}\right)_{n \geq 1}$ denote a sequence of real-valued random variables on a probability space $(\Omega, \mathcal{F}, P)$. No dependence between the variables is assumed. For $-\pi \leq t<\pi$, define the discrete Fourier transform

$$
S_{n}(t)=\sum_{k=1}^{n} e^{i k t} X_{k}
$$

We shall establish an anologue of the result by Baum and Katz (1965) for the discrete Fourier transform.

Theorem 2.1. Assume

$$
P\left(\left|X_{n}\right|>x\right) \leq P\left(\left|X_{1}\right|>x\right), \quad \text { for all } x \geq 0, \quad n \geq 1 .
$$

If $E\left|X_{1}\right|<\infty$, then for almost all $t \in[-\pi, \pi)$,

$$
\lim _{n \rightarrow \infty} \frac{S_{n}(t)}{n}=0, \quad P-\text { a.s. }
$$

The following theorem describes the rate of convergence in the strong law of large numbers.

Theorem 2.2. Assume

$$
\frac{1}{n} \sum_{k=1}^{n} P\left(\left|X_{k}\right|>x\right) \leq M P\left(\left|X_{1}\right|>x\right), \quad \text { for all } x \geq 0, \quad n \geq 1
$$

Let $1<p<2,1 \leq r \leq p$. If $E\left|X_{1}\right|^{p}<\infty$, then for every $\epsilon>0$ and for almost all $t \in[-\pi, \pi)$,

$$
\sum_{n=1}^{\infty} n^{p / r-2} P\left[\max _{1 \leq k \leq n}\left|S_{k}(t)\right|>\epsilon n^{1 / r}\right]<\infty .
$$

Remark 2.3. In fact, Gut (1992) gave an example to show that condition (MD) is strictly weaker than condition (UD). But in Theorem 2.1, it is an open question whether condition (UD) can be weakened to condition (MD).

Remark 2.4. Differently from the i.i.d. case, the reciprocal of Theorem 2.2 is no longer true. That is, (2) does not imply $X_{1}$ have finite $p t h$ moment.

Corollary 2.5. Under the assumption of Theorem 2.2, for almost all $t \in[\pi, \pi)$,

$$
\lim _{n \rightarrow \infty} \frac{S_{n}(t)}{n^{1 / p}}=0, \quad P-a . s .
$$

Remark 2.6. Both theorems hold if the variables have the same distribution. 


\section{Proofs}

Throughout this whole paper, $C>0$ denotes a generic constant which may take different values from line to line.

In order to prove our main theorems, we shall first establish two preparatory lemmas. We begin by a truncation argument.

Lemma 3.1. Assume $\left(X_{n}\right)_{n \geq 1}$ satisfies condition (UD) and $E\left|X_{1}\right|<\infty$. Let $Y_{k}=X_{k} I\left\{\left|X_{k}\right| \leq k\right\}$ and $S_{n}^{*}(t)=\sum_{k=1}^{n} e^{i k t} Y_{k}$. Then for all $t$ in $[-\pi, \pi)$,

$$
\lim _{n \rightarrow \infty}\left|\frac{1}{n} S_{n}(t)-\frac{1}{n} S_{n}^{*}(t)\right|=0, \quad P-\text { a.s. }
$$

Proof. As $P\left(\left|X_{n}\right|>x\right) \leq P\left(\left|X_{1}\right|>x\right)$, we obtain

$$
\sum_{n=1}^{\infty} P\left(X_{n} \neq Y_{n}\right)=\sum_{n=1}^{\infty} P\left(\left|X_{n}\right|>n\right) \leq \sum_{n=1}^{\infty} P\left(\left|X_{1}\right|>n\right) \leq \int_{0}^{\infty} P\left(\left|X_{1}\right|>x\right) d x=E\left|X_{1}\right|<\infty .
$$

By the Borel-Cantelli Lemma, we know $P\left(X_{n} \neq Y_{n}\right.$ i.o. $)=0$. That is, for almost all $\omega \in \Omega$, $X_{n}(\omega)=Y_{n}(\omega)$, for all $n$ sufficiently large, say for all $n \geq m(\omega):=m$. Thus for all $t \in[-\pi, \pi)$,

$\left|\frac{1}{n} S_{n}(t)-\frac{1}{n} S_{n}^{*}(t)\right|=\frac{1}{n}\left|\sum_{k=1}^{n} e^{i k t}\left(X_{k}-Y_{k}\right)\right| \leq \frac{1}{n} \sum_{k=1}^{m}\left|e^{i k t}\right| \cdot\left|X_{k}-Y_{k}\right|=\frac{1}{n} \sum_{k=1}^{m}\left|X_{k}-Y_{k}\right| \rightarrow 0, \quad P-$ a.s.

That is,

$$
\lim _{n \rightarrow \infty}\left|\frac{1}{n} S_{n}(t)-\frac{1}{n} S_{n}^{*}(t)\right|=0, \quad P-\text { a.s. }
$$

Lemma 3.2. Assume $\left(X_{n}\right)_{n \geq 1}$ satisfies condition (MD) and $E\left|X_{1}\right|^{p}<\infty$. Then

$$
\begin{aligned}
& \sum_{n=1}^{\infty} n^{p / r-1 / r-2} \sum_{k=1}^{n} E\left[\left|X_{k}\right| I\left(\left|X_{k}\right|>n^{1 / r}\right)\right] \leq C E\left|X_{1}\right|^{p}<\infty \\
& \sum_{n=1}^{\infty} n^{p / r-2 / r-2} \sum_{k=1}^{n} E\left[\left|X_{k}\right|^{2} I\left(\left|X_{k}\right| \leq n^{1 / r}\right)\right] \leq C E\left|X_{1}\right|^{p}<\infty .
\end{aligned}
$$

Proof. We shall use the following facts: For $X \geq 0$ a.s. and $A>0$,

$$
E(X)=\int_{0}^{\infty} P(X>x) d x ; \quad E(X I(X>A))=A P(X>A)+\int_{A}^{\infty} P(X>x) d x
$$

and

$$
E(X I(X \leq A))=-A P(X>A)+\int_{0}^{A} P(X>x) d x \leq \int_{0}^{A} P(X>x) d x .
$$

Therefore

$$
E\left(X^{2} I\left(X^{2} \leq A\right)\right) \leq \int_{0}^{A} P\left(X^{2}>x\right) d x
$$


As $\left(X_{n}\right)_{n \geq 1}$ satisfies condition (MD), by fact (5), we get

$$
\sum_{k=1}^{n} E\left[\left|X_{k}\right| I\left(\left|X_{k}\right|>n^{1 / r}\right)\right] \leq M n E\left[\left|X_{1}\right| I\left(\left|X_{1}\right|>n^{1 / r}\right)\right] .
$$

So then

$$
\sum_{n=1}^{\infty} n^{p / r-1 / r-2} \sum_{k=1}^{n} E\left[\left|X_{k}\right| I\left(\left|X_{k}\right|>n^{1 / r}\right)\right] \leq M \sum_{n=1}^{\infty} n^{p / r-1 / r-1} E\left[\left|X_{1}\right| I\left(\left|X_{1}\right|>n^{1 / r}\right)\right] .
$$

By Stoica (2011) (page 912), we have

$$
\sum_{n=1}^{\infty} n^{p / r-1 / r-1} E\left[\left|X_{1}\right| I\left(\left|X_{1}\right|>n^{1 / r}\right)\right] \leq C E\left|X_{1}\right|^{p}<\infty .
$$

Combining this result with our computation, we obtain (3).

By condition (MD) and (7), we get

$$
\begin{aligned}
\sum_{k=1}^{n} E X_{k}^{2} I\left(\left|X_{k}\right| \leq n^{1 / r}\right) & \leq \sum_{k=1}^{n} \int_{0}^{n^{2 / r}} P\left(X_{k}^{2}>x\right) d x \\
& \leq n M \int_{0}^{n^{2 / r}} P\left(X_{1}^{2}>x\right) d x \\
& \leq C n \sum_{k=1}^{n^{2 / r}} P\left(X_{1}^{2}>k\right) .
\end{aligned}
$$

Now

$$
\begin{aligned}
\sum_{n=1}^{\infty} n^{p / r-2 / r-2} n \sum_{k=1}^{n^{2 / r}} P\left(X_{1}^{2}>k\right) & \leq \sum_{k=1}^{\infty} P\left(X_{1}^{2}>k\right) \sum_{n \geq k^{r / 2}} n^{p / r-2 / r-1} \\
& \leq C \sum_{k=1}^{\infty} P\left(X_{1}^{2}>k\right) k^{\frac{p-2}{2}} \leq C E\left|X_{1}\right|^{p}
\end{aligned}
$$

because

$$
E\left|X_{1}\right|^{p}=E\left[\left(X_{1}^{2}\right)^{p / 2}\right]=\frac{p}{2} \int_{0}^{\infty} x^{\frac{p-2}{2}} P\left(X_{1}^{2}>x\right) d x .
$$

Therefore we have (4).

\subsection{Proof of Theorem 2.1}

Proof. First, let us show that $\sum_{k=1}^{n} \frac{e^{i k t} Y_{k}}{k}$ converges a.s. for almost all $t \in[-\pi, \pi)$. 
From Durrett (2013) (page 64), we know that $\sum_{k=1}^{\infty} \frac{E Y_{k}^{2}}{k^{2}} \leq 4 E\left|X_{1}\right|<\infty$. So, for almost all $\omega \in \Omega$, $\sum_{k=1}^{\infty} \frac{Y_{k}^{2}(\omega)}{k^{2}}<\infty$

Then, by Carleson's Theorem (1966), for almost all $\omega \in \Omega, \sum_{k=1}^{n} \frac{e^{i k t} Y_{k}(\omega)}{k}$ converges almost everywhere in $t$. That is, for almost all $\omega \in \Omega$, there exists $I_{\omega} \subset[-\pi, \pi)$ with $\lambda\left(I_{\omega}\right)=2 \pi$, such that for all $t \in I_{\omega}, \sum_{k=1}^{n} \frac{e^{i k t} Y_{k}(\omega)}{k}$ converges, where $\lambda$ is the Lebesgue measure on $[-\pi, \pi)$.

Let $\Omega_{0}=\left\{\omega: \sum_{k=1}^{\infty} \frac{Y_{k}^{2}(\omega)}{k^{2}}<\infty\right\}$, then $P\left(\Omega_{0}\right)=1$.

It is convenient to work on the product space $[-\pi, \pi) \times \Omega$ with product measure $\widetilde{P}:=\lambda \times P$.

Define $A=\left\{(t, \omega): \sum_{k=1}^{\infty} \frac{e^{i k t} Y_{k}(\omega)}{k}\right.$ is convergent $\} \subset[-\pi, \pi) \times \Omega$.

Using Fubini Theorem,

$$
\begin{aligned}
\widetilde{P}(A) & =\int_{[-\pi, \pi) \times \Omega} I_{A}(t, \omega) d \widetilde{P}=\int_{\Omega} \int_{-\pi}^{\pi} I_{A}(t, \omega) \lambda(d t) d P \\
& =\int_{\Omega_{0}} \int_{-\pi}^{\pi} I_{A}(t, \omega) \lambda(d t) d P=\int_{\Omega_{0}} 2 \pi d P \\
& =2 \pi \\
& =\int_{-\pi}^{\pi} \int_{\Omega} I_{A}(t, \omega) d P \lambda(d t) .
\end{aligned}
$$

Thus, for almost all $t \in[-\pi, \pi), \sum_{k=1}^{n} \frac{e^{i k t} Y_{k}}{k}$ converges a.s.

Now by applying Kronecker Lemma (If $a_{n} \uparrow \infty$ and $\sum_{n=1}^{\infty}\left(x_{n} / a_{n}\right)$ converges, then $a_{n}^{-1} \sum_{m=1}^{n} x_{m} \rightarrow$ 0 ), for almost all $t \in[-\pi, \pi)$, we obtain

$$
\lim _{n \rightarrow \infty} \frac{1}{n} \sum_{k=1}^{n} e^{i k t} Y_{k}=\lim _{n \rightarrow \infty} \frac{1}{n} S_{n}^{*}(t)=0, \quad P-\text { a.s. }
$$

By Lemma 3.1, it follows that for almost all $t \in[-\pi, \pi)$,

$$
\lim _{n \rightarrow \infty} \frac{1}{n} S_{n}(t)=\lim _{n \rightarrow \infty} \frac{1}{n} S_{n}^{*}(t)=0, \quad P-\text { a.s. }
$$

\subsection{Proof of Theorem 2.2}

Proof. Define the following random variables for $k=1,2, \ldots, n$ :

$$
X_{k}^{\prime}=e^{i t k} X_{k} I\left\{\left|X_{k}\right| \leq n^{1 / r}\right\}, \quad X_{k}^{\prime \prime}=e^{i t k} X_{k} I\left\{\left|X_{k}\right|>n^{1 / r}\right\} .
$$

Clearly, $e^{i t k} X_{k}=X_{k}^{\prime}+X_{k}^{\prime \prime}$ and $S_{n}(t)=S_{n}^{\prime}(t)+S_{n}^{\prime \prime}(t)$ where $S_{n}^{\prime}(t)=\sum_{k=1}^{n} X_{k}^{\prime}$ and $S_{n}^{\prime \prime}(t)=\sum_{k=1}^{n} X_{k}^{\prime \prime}$. 
By Markov's Inequality,

$$
P\left[\max _{1 \leq k \leq n}\left|S_{k}^{\prime}(t)\right|>\epsilon n^{1 / r}\right] \leq \frac{1}{\epsilon^{2}} n^{-2 / r} E\left[\left(\max _{1 \leq k \leq n}\left|S_{k}^{\prime}(t)\right|\right)^{2}\right],
$$

and by the maximal inequality in Hunt and Young (1974),

$$
\int_{-\pi}^{\pi} \max _{1 \leq k \leq n}\left|S_{k}^{\prime}(t)\right|^{2} \lambda(d t) \leq C \sum_{k=1}^{n}\left|X_{k}^{\prime}\right|^{2} .
$$

Using Fubini's Theorem and properties (8) and (9), we obtain

$$
\begin{aligned}
\widetilde{P}\left[\max _{1 \leq k \leq n}\left|S_{k}^{\prime}(t)\right|>\epsilon n^{1 / r}\right] & =\int_{-\pi}^{\pi} P\left[\max _{1 \leq k \leq n}\left|S_{k}^{\prime}(t)\right|>\epsilon n^{1 / r}\right] \lambda(d t) \\
& \leq \frac{1}{\epsilon^{2}} n^{-2 / r} \int_{-\pi}^{\pi} E\left[\left(\max _{1 \leq k \leq n}\left|S_{k}^{\prime}(t)\right|\right)^{2}\right] \lambda(d t) \\
& \leq \frac{C}{\epsilon^{2}} n^{-2 / r} E\left[\sum_{k=1}^{n}\left|X_{k}^{\prime}\right|^{2}\right] \\
& =\frac{C}{\epsilon^{2}} n^{-2 / r} \sum_{k=1}^{n} E\left[\left(X_{k}\right)^{2} I\left(\left|X_{k}\right| \leq n^{1 / r}\right)\right] .
\end{aligned}
$$

By Lemma 3.2, we get

$$
\sum_{n=1}^{\infty} n^{p / r-2} \widetilde{P}\left[\max _{1 \leq k \leq n}\left|S_{k}^{\prime}(t)\right|>\epsilon n^{1 / r}\right]<\infty
$$

By Markov's inequality,

$$
\begin{aligned}
P\left[\max _{1 \leq k \leq n}\left|S_{k}^{\prime \prime}(t)\right|>\epsilon n^{1 / r}\right] & \leq \frac{1}{\epsilon} n^{-1 / r} E\left[\max _{1 \leq k \leq n}\left|S_{k}^{\prime \prime}(t)\right|\right] \\
& \leq \frac{1}{\epsilon} n^{-1 / r} E\left[\sum_{k=1}^{n}\left|X_{k}^{\prime \prime}\right|\right] \\
& =\frac{1}{\epsilon} n^{-1 / r} \sum_{k=1}^{n} E\left[\left|X_{k}\right| I\left\{\left|X_{k}\right|>n^{1 / r}\right\}\right] .
\end{aligned}
$$

Again, by Lemma 3.2, we obtain

$$
\sum_{n=1}^{\infty} n^{p / r-2} P\left[\max _{1 \leq k \leq n}\left|S_{k}^{\prime \prime}(t)\right|>\epsilon n^{1 / r}\right] \leq C E\left|X_{1}\right|^{p}<\infty .
$$

Using Fubini's Theorem and relation (11),

$$
\begin{aligned}
\sum_{n=1}^{\infty} n^{p / r-2} \widetilde{P}\left[\max _{1 \leq k \leq n}\left|S_{k}^{\prime \prime}(t)\right|>\epsilon n^{1 / r}\right] & =\sum_{n=1}^{\infty} n^{p / r-2} \int_{-\pi}^{\pi} P\left[\max _{1 \leq k \leq n}\left|S_{k}^{\prime \prime}(t)\right|>\epsilon n^{1 / r}\right] \lambda(d t) \\
& \leq C E\left|X_{1}\right|^{p}<\infty
\end{aligned}
$$


Combining (10) and (12), we get

$$
\begin{aligned}
& \sum_{n=1}^{\infty} n^{p / r-2} \widetilde{P}\left[\max _{1 \leq k \leq n}\left|S_{k}(t)\right|>\epsilon n^{1 / r}\right] \\
& \leq \sum_{n=1}^{\infty} n^{p / r-2} \widetilde{P}\left[\max _{1 \leq k \leq n}\left|S_{k}^{\prime}(t)\right|>\frac{\epsilon}{2} n^{1 / r}\right]+\sum_{n=1}^{\infty} n^{p / r-2} \widetilde{P}\left[\max _{1 \leq k \leq n}\left|S_{k}^{\prime \prime}(t)\right|>\frac{\epsilon}{2} n^{1 / r}\right]<\infty .
\end{aligned}
$$

By Fubini's Theorem, we have

$$
\begin{aligned}
& \sum_{n=1}^{\infty} n^{p / r-2} \widetilde{P}\left[\max _{1 \leq k \leq n}\left|S_{k}(t)\right|>\epsilon n^{1 / r}\right] \\
& =\sum_{n=1}^{\infty} n^{p / r-2} \int_{-\pi}^{\pi} \int_{\Omega} I\left\{\max _{1 \leq k \leq n}\left|S_{k}(t)\right|>\epsilon n^{1 / r}\right\} d P \lambda(d t) \\
& =\int_{-\pi}^{\pi} \sum_{n=1}^{\infty} n^{p / r-2} P\left(\max _{1 \leq k \leq n}\left|S_{k}(t)\right|>\epsilon n^{1 / r}\right) \lambda(d t)<\infty .
\end{aligned}
$$

Thus, for almost all $t \in[-\pi, \pi)$,

$$
\sum_{n=1}^{\infty} n^{p / r-2} P\left(\max _{1 \leq k \leq n}\left|S_{k}(t)\right|>\epsilon n^{1 / r}\right)<\infty .
$$

\subsection{Proof of Remark 2.4}

Proof. It is well-known that $E\left|X_{1}\right|^{p}<\infty$ is equivalent to (2) in the i.i.d. case, but this is no longer true in our setting.

For instance, let

$$
X_{1}=X_{2}=\cdots=X_{n}=\cdots .
$$

Then

$$
\max _{1 \leq k \leq n}\left|S_{k}(t)\right|=\max _{1 \leq k \leq n}\left|\sum_{j=1}^{k} e^{i j t} X_{k}\right|=\max _{1 \leq k \leq n}\left|\frac{1-e^{i k t}}{1-e^{i t}}\right| \cdot\left|X_{1}\right| .
$$

Because

$$
1 \leq \max _{1 \leq k \leq n}\left|\frac{1-e^{i k t}}{1-e^{i t}}\right| \leq \frac{2}{\left|1-e^{i t}\right|},
$$

(2) is equivalent to

$$
\sum_{n=1}^{\infty} n^{p / r-2} P\left(\left|X_{1}\right|>C \epsilon n^{1 / r}\right)<\infty,
$$

which is certainly not equivalent to $E\left|X_{1}\right|^{p}<\infty$. 


\subsection{Proof of Corollary 2.5}

Proof. By Theorem 2.2, when $1<r=p<2$, we have: for almost all $t \in[-\pi, \pi)$,

$$
\sum_{n=1}^{\infty} n^{-1} P\left(\max _{1 \leq k \leq n}\left|S_{k}(t)\right|>\epsilon n^{1 / p}\right)<\infty
$$

which is equivalent to

$$
\sum_{N=1}^{\infty} P\left(\max _{1 \leq k \leq 2^{N}}\left|S_{k}(t)\right|>\epsilon 2^{N / p}\right)<\infty
$$

Then, by the Borel-Cantelli Lemma, for almost all $t \in[-\pi, \pi)$,

$$
S_{n}(t) / n^{1 / p} \rightarrow 0, \quad P-\text { a.s. }
$$

\section{Acknowledgement}

The author's research was partially supported by the NSF grant, DMS-1512936.

The author is indebted to to Magda Peligrad for her instructions to write the paper and to the referees for carefully reading the paper and for numerous suggestions which improved the presentation of the paper.

\section{References}

[1] Baum, L.E. and Katz, M., Convergence rates in the law of large numbers, Trans.Amer.Math.Soc. 120 (1965), pp. 108-123.

[2] Billingsley, P., Probability and Measure, Anniversary ed., Wiley, 2012.

[3] Carleson, L., On convergence and growth of partial sums of Fourier series, Acta Mathematica, 116 (1966), no.1, pp. 135-157.

[4] Dedecker, J. and Merlevede, F., Convergence rates in the law of large numbers for Banach-valued dependent variables, Theory Probab. Appl. 52 (2008), pp. 416-438.

[5] Durrett, R., Probability: Theory and Examples, 4th ed., Cambridge, NewYork, 2013.

[6] Etemadi, N., An elementary proof of the strong law of large numbers, Z. Wahrsch. Verw. Gebiete 55 (1981), no. 1, pp. 119-122.

[7] Gut, A., Complete convergence for arrays, Period. Math. Hungar. 25 (1992), no. 1, pp. 51-75.

[8] Hunt, R.A. and Young, W.S., A weighted norm inequality for Fourier Series, Bull.Amer.Math. 80 (1974), pp. 274-277.

[9] Korchevsky, V., Marcinkiewicz-Zygmund Strong law of large numbers for pairwise i.i.d. random variables, Aug. 2015, arXiv: 1404.7454 [math.PR].

[10] Marcinkiewicz, J. and Zygmund, A., Sur les fonctions indépendantes, Fund.Math. 29 (1937), pp. 60-90.

[11] Stoica, G., A note on the rate of convergence in the strong law of large numbers for martingales, J.Math. Anal. Appl. 381 (2011), pp. 910-913. 